\title{
ON EXISTENCE OF A SOLUTION FOR THE SYSTEM OF GENERALIZED VECTOR QUASI-EQUILIBRIUM PROBLEMS WITH UPPER SEMICONTINUOUS SET-VALUED MAPS
}

\author{
JIANWEN PENG AND XINMIN YANG
}

Received 28 September 2004 and in revised form 7 July 2005

We introduce a new model of the system of generalized vector quasi-equilibrium problems with upper semicontinuous set-valued maps and present several existence results of a solution for this system of generalized vector quasi-equilibrium problems and its special cases. The results in this paper extend and improve some results in the literature.

\section{Introduction and preliminaries}

Throughout this paper, we use int $A$ and $\operatorname{Co} A$ to denote the interior and the convex hull of a set $A$, respectively.

Let $I$ be an index set. For each $i \in I$, let $Y_{i}, E_{i}$ be two Hausdorff topological vector spaces. Consider a family of nonempty convex subsets $\left\{X_{i}\right\}_{i \in I}$ with $X_{i} \subseteq E_{i}$. Let $X=$ $\prod_{i \in I} X_{i}$ and $E=\prod_{i \in I} E_{i}$. An element of the set $X^{i}=\prod_{j \in I \backslash i} X_{i}$ will be denoted by $x^{i}$; therefore, $x \in X$ will be written as $x=\left(x^{i}, x_{i}\right) \in X^{i} \times X_{i}$. For each $i \in I$, let $D_{i}: X \rightarrow 2^{X_{i}}$ and $F_{i}: X \times X_{i} \rightarrow 2^{Y_{i}}$ be two set-valued maps, let $C_{i}: X \rightarrow 2^{Y_{i}}$ be a set-valued map such that $C_{i}(x)$ is a convex, pointed, and closed cone with int $C_{i}(x) \neq \varnothing$ for all $x \in X$. Then the system of generalized vector quasi-equilibrium problems with set-valued maps (in short, SGVQEP) is to find $\bar{x}=\left(\bar{x}^{i}, \bar{x}_{i}\right)$ in $X$ such that for each $i \in I$,

$$
\bar{x}_{i} \in D_{i}(\bar{x}), \quad F_{i}\left(\bar{x}, y_{i}\right) \nsubseteq-\operatorname{int} C_{i}(\bar{x}), \quad \forall y_{i} \in D_{i}(\bar{x}) .
$$

Remark 1.1. In [32], we introduced and studied another type of system of generalized vector quasi-equilibrium problems with lower semicontinous set-valued maps, which is to find $\bar{x}=\left(\bar{x}^{i}, \bar{x}_{i}\right)$ in $X$ such that for each $i \in I$,

$$
\bar{x}_{i} \in D_{i}(\bar{x}), \quad F_{i}\left(\bar{x}, y_{i}\right) \subseteq Y_{i} \backslash\left(-\operatorname{int} C_{i}(\bar{x})\right), \quad \forall y_{i} \in D_{i}(\bar{x}) .
$$

It is apparent that this problem is different from the SGVQEP.

The following problems are special cases of the SGVQEP.

(1) For each $i \in I$ and for all $x \in X$, if $D_{i}(x) \equiv X_{i}$, then the SGVQEP reduces to the system of generalized vector equilibrium problems with set-valued maps (in short, SGVEP) 
which is to find $\bar{x}$ in $X$ such that for each $i \in I$,

$$
F_{i}\left(\bar{x}, y_{i}\right) \nsubseteq-\operatorname{int} C_{i}(\bar{x}), \quad \forall y_{i} \in X_{i}
$$

This problem was introduced and studied by Ansari et al. in [20].

(2) For each $i \in I$, if the set-valued map $F_{i}$ is replaced by a vector-valued map $\varphi_{i}$ : $X \times X_{i} \rightarrow Y_{i}$, then the SGVQEP reduces to a system of vector quasi-equilibrium problems (in short, SVQEP) which is to find $\bar{x}=\left(\bar{x}^{i}, \bar{x}_{i}\right)$ in $X$ such that for each $i \in I$,

$$
\bar{x}_{i} \in D_{i}(\bar{x}), \quad \varphi_{i}\left(\bar{x}, y_{i}\right) \notin-\operatorname{int} C_{i}(\bar{x}), \quad \forall y_{i} \in D_{i}(\bar{x}) .
$$

For each $i \in I$, let $\varphi_{i}: X \rightarrow Y_{i}$ be a vector-valued map, and let $f_{i}\left(x, y_{i}\right)=\varphi_{i}\left(x^{i}, y_{i}\right)-$ $\varphi_{i}(x)$, then the SVQEP is equivalent to the following Debreu-type equilibrium problem for vector-valued maps (in short, Debreu VEP) which is to find $\bar{x}=\left(\bar{x}^{i}, \bar{x}_{i}\right)$ in $X$ such that for each $i \in I$,

$$
\bar{x}_{i} \in D_{i}(\bar{x}), \quad \varphi_{i}\left(\bar{x}^{i}, y_{i}\right)-\varphi_{i}(\bar{x}) \notin-\operatorname{int} C_{i}(\bar{x}), \quad \forall y_{i} \in D_{i}(\bar{x}) .
$$

The SVQEP and the Debreu VEP were introduced and studied by Ansari et al. [21]. And if $D_{i}(x) \equiv X_{i}$ for each $i \in I$ and for all $x \in X$, then the Debreu VEP becomes the Nash equilibrium problem for vector-valued maps in [19].

If $Y_{i}=R, C_{i}(x)=\{y \in R \mid y \leq 0\}$ for each $i \in I$ and for all $x \in X$, and $\varphi_{i}$ is a scalar realvalued function from $X \times X_{i}$ to $R$, then the SVQEP reduces to the model of generalized game in [22, page 286] and quasivariational inequalities in [23, page 152-153].

(3) For each $i \in I$, let $\eta_{i}: X_{i} \times X_{i} \rightarrow E_{i}$ be a single-valued map and $T_{i}: X \rightarrow 2^{L\left(E_{i}, Y_{i}\right)}$ a set-valued map, where $L\left(E_{i}, Y_{i}\right)$ denotes the space of all continuous linear operators from $E_{i}$ into $Y_{i}$. Let $F_{i}\left(x, y_{i}\right)=\left\langle T_{i}(x), \eta_{i}\left(y_{i}, x_{i}\right)\right\rangle=\bigcup_{v_{i} \in T_{i}(x)}\left\langle v_{i}, \eta_{i}\left(y_{i}, x_{i}\right)\right\rangle$. Then the SGVQEP reduces to the system of generalized vector quasivariational-like inequality problems (in short, SGVQVLIP), which is to find $\bar{x}=\left(\bar{x}^{i}, \bar{x}_{i}\right)$ in $X$ such that for each $i \in I$,

$$
\bar{x}_{i} \in D_{i}(\bar{x}), \quad \forall y_{i} \in D_{i}(\bar{x}), \quad \exists \bar{v}_{i} \in T_{i}(\bar{x}):\left\langle\bar{v}_{i}, \eta_{i}\left(y_{i}, \bar{x}_{i}\right)\right\rangle \notin-\operatorname{int} C_{i}(\bar{x}) .
$$

For each $i \in I$, let $\eta_{i}\left(y_{i}, x_{i}\right)=y_{i}-x_{i}$ for all $x_{i}, y_{i} \in X_{i}$, then the SGVQVLIP reduces to the system of generalized vector quasivariational inequality problems (in short, SGVQVIP) which is to find $\bar{x}=\left(\bar{x}^{i}, \bar{x}_{i}\right)$ in $X$ such that for each $i \in I, \bar{x}_{i} \in D_{i}(\bar{x})$,

$$
\forall y_{i} \in D_{i}(\bar{x}), \quad \exists \bar{v}_{i} \in T_{i}(\bar{x}):\left\langle\bar{v}_{i}, y_{i}-\bar{x}_{i}\right\rangle \notin-\operatorname{int} C_{i}(\bar{x}) .
$$

The SGVQVLIP and the SGVQVIP were introduced by Peng [18].

For each $i \in I$, if $D_{i}(x) \equiv X_{i}$ for all $x \in X$, then the SGVQVLIP reduces to the system of generalized vector variational-like inequality problems (in short, SGVVLIP) which is to find $\bar{x}=\left(\bar{x}^{i}, \bar{x}_{i}\right)$ in $X$ such that for each $i \in I$,

$$
\forall y_{i} \in X_{i}, \quad \exists \bar{v}_{i} \in T_{i}(\bar{x}):\left\langle\bar{v}_{i}, \eta_{i}\left(y_{i}, x_{i}\right)\right\rangle \notin-\operatorname{int} C_{i}(\bar{x}) .
$$

For each $i \in I$, if $D_{i}(x) \equiv X_{i}$ for all $x \in X$, and $\eta_{i}\left(y_{i}, x_{i}\right)=y_{i}-x_{i}$ for all $x_{i}, y_{i} \in X_{i}$, then the SGVQVLIP reduces to the system of generalized vector variational inequality 
problems (in short, SGVVIP) which is to find $\bar{x}=\left(\bar{x}^{i}, \bar{x}_{i}\right)$ in $X$ such that

$$
\forall y_{i} \in X_{i}, \quad \exists \bar{v}_{i} \in T_{i}(\bar{x}):\left\langle\bar{v}_{i}, y_{i}-\bar{x}_{i}\right\rangle \notin-\operatorname{int} C_{i}(\bar{x}) .
$$

The SGVVLIP and the SGVVIP were introduced by Ansari et al. in [20].

For each $i \in I$, if $Y_{i} \equiv Y$ and $C_{i}(x) \equiv C$ for all $x \in X$, where $C$ is a convex, closed, and pointed cone in $Y$ with int $C \neq \varnothing$, then the SGVVIP reduces to another kind of system of generalized vector variational inequality problems (in short, II-SGVVIP) which is to find $\bar{x}=\left(\bar{x}^{i}, \bar{x}_{i}\right)$ in $X$ such that

$$
\forall y_{i} \in X_{i}, \quad \exists \bar{v}_{i} \in T_{i}(\bar{x}):\left\langle\bar{v}_{i}, y_{i}-\bar{x}_{i}\right\rangle \notin-\operatorname{int} C .
$$

This was studied by Allevi et al. [17].

If $T_{i}$ is a single-valued function, then the II-SGVVIP reduces to the system of vector variational inequality problems (in short, SVVIP), which is to find $\bar{x}=\left(\bar{x}^{i}, \bar{x}_{i}\right)$ in $X$ such that

$$
\left\langle T_{i}(\bar{x}), y_{i}-\bar{x}_{i}\right\rangle \notin-\operatorname{int} C, \quad \forall y_{i} \in X_{i} .
$$

This was considered by Ansari et al. in [19].

Let $Y=R$ and $C=R^{+}=\{r \in R: r \geq 0\}$. For each $i \in I$, if $T_{i}$ is replaced by $f_{i}: X \rightarrow R$, then the SVVIP reduces to the system of scalar variational inequality problems which is to find $\bar{x}=\left(\bar{x}^{i}, \bar{x}_{i}\right)$ in $X$ such that

$$
\left\langle f_{i}(\bar{x}), y_{i}-\bar{x}_{i}\right\rangle \geq 0, \quad \forall y_{i} \in X_{i}
$$

This was considered in $[16,24,25,26]$.

(4) If the index set $I$ is singleton, then the SGVQEP reduces to the generalized vector quasi-equilibrium problem (in short, GVQEP) studied in $[12,15]$ which contains the generalized vector equilibrium problems studied in $[27,28,29]$ as special cases, and the SGVVLIP reduces to the generalized vector variational-like inequality problem studied by Ding and Tarafdar [31].

In this paper, we present some existence results of a solution for the SGVQEP and its special cases with or without $\Phi$-condensing maps.

Now we introduce a new definition as follows.

Definition 1.2. Let $I$ be an index set. For each $i \in I$, let $Y_{i}$ be a topological vector space and $X_{i}$ a nonempty convex subset of a Hausdorff topological vector space $E_{i}$, and $F_{i}: X \times X_{i} \rightarrow$ $2^{Y_{i}}$ be a set-valued map, let $C_{i}: X \rightarrow 2^{Y_{i}}$ be a set-valued map such that $C_{i}(x)$ be a convex and closed cone with int $C_{i}(x) \neq \varnothing$ for all $x \in X_{i} . F_{i}\left(x, z_{i}\right)$ is said to be $C_{i-x}$-0-partially diagonal quasiconvex if for any finite set $\left\{y_{i_{1}}, y_{i_{2}}, \ldots, y_{i_{n}}\right\}$ in $X_{i}$, and for all $x=\left(x^{i}, x_{i}\right) \in X$ with $x_{i} \in \operatorname{Co}\left\{y_{i_{1}}, y_{i_{2}}, \ldots, y_{i_{n}}\right\}$, there exists some $j(j=1,2, \ldots, n)$ such that

$$
F_{i}\left(x, y_{i_{j}}\right) \nsubseteq-\operatorname{int} C_{i}(x) .
$$

Remark 1.3. (a) If the formula (1.13) is replaced by $F_{i}\left(x, y_{i_{j}}\right) \subseteq Y_{i} \backslash\left(-\operatorname{int} C_{i}(x)\right)$, then Definition 1.2 becomes [32, Definition 1.1], which is called to be the second type $C_{i-x}-0$ partially diagonal quasiconvexity of $F_{i}$ in this paper. 


\section{System of generalized vector quasi-equilibrium problems}

(b) For each $i \in I$, if the set-valued map $F_{i}: X \times X_{i} \rightarrow 2^{Y_{i}}$ is replaced by a singlevalued map $f_{i}: X \times X_{i} \rightarrow Y_{i}$ and (1.13) is replaced by $f_{i}\left(x, y_{i_{j}}\right) \notin-\operatorname{int} C_{i}(x)$, then the $C_{i-x}$-0-partially diagonal quasiconvexity of $F_{i}$ reduces to the $C_{i-x}-0$-partially diagonal quasiconvexity of $f_{i}$. Furthermore, if $C_{i}(x)=C_{i}$, then the $C_{i-x}-0$-partially diagonal quasiconvexity of $f_{i}$ reduces to the $C_{i}$-0-partially diagonal quasiconvexity of $f_{i}$. If $Y_{i}=R$ and $C_{i}=\{r \in R: r \geq 0\}$ for each $i \in I$, then the $C_{i}$-0-partially diagonal quasiconvexity of $f_{i}$ reduces to [30, Definition 3]. Furthermore, let $I=\{1\}$, then [30, Definition 3] reduces to the $\gamma$-diagonal quasiconvexity in [3], here $\gamma=0$.

(c) For each $i \in I$, let $E_{i}$ be a real normed space with dual space $E_{i}{ }^{*}, X_{i} \subset E_{i}, Z_{i}=R$. Let $\|\bullet\|_{i}$ denote the norm on $E_{i}$. If we define a norm on $E$ as

$$
\|x\|=\sum_{i=1}^{n}\left\|x_{i}\right\|_{i}, \quad \forall x=\left(x_{1}, x_{2}, \ldots, x_{n}\right) \in E,
$$

then it is easy to verify that $\|\bullet\|$ is a norm on $E$. And hence $E$ is also a real normed space. Let $C_{i}: X \rightarrow 2^{Z_{i}}$ be defined as $C_{i}(x)=[\|x\|,+\infty)$, for all $x \in X$, and let $\left[e_{1}, e_{2}\right]$ denote the line segment joining $e_{1}$ and $e_{2}$. Choosing $p_{i} \in E_{i}{ }^{*}$, we define a set-valued map $F$ : $X \times X_{i} \rightarrow 2^{Z_{i}}$ as

$$
F\left(x, z_{i}\right)=\left\{\left\langle u, z_{i}-x_{i}\right\rangle: u \in\left[-2\|x\|\left\|z_{i}\right\|_{i} p_{i},-\|x\|\left\|z_{i}\right\|_{i} p_{i}\right]\right\}, \quad \forall\left(x, z_{i}\right) \in X \times X_{i},
$$

Then, $F$ is $C_{i-x}-0$-partially diagonal quasiconvex in the second argument. Otherwise, there exists finite set $\left\{z_{i_{1}}, z_{i_{2}}, \ldots, z_{i_{n}}\right\} \subseteq X_{i}$, and there is $x \in X$ with $x_{i}=\sum_{j=1}^{n} \alpha_{j} z_{i_{j}}\left(\alpha_{j} \geq\right.$ $\left.0, \sum_{j=1}^{n} \alpha_{j}=1\right)$ such that for all $j=1,2, \ldots, n, F\left(x, z_{i_{j}}\right) \subseteq-\operatorname{int} C_{i}(x)$. Then for each $j$, for all $\lambda_{j} \in[0,1]$, we have

$$
\left\langle\lambda_{j}\left(-2\|x\|\left\|z_{i_{j}}\right\|_{i} p_{i}\right)+\left(1-\lambda_{j}\right)\left(-\|x\|\left\|z_{i_{j}}\right\|_{i} p_{i}\right), z_{i_{j}}-x_{i}\right\rangle<-\|x\| \leq 0 .
$$

It follows that

$$
\left\langle p_{i}, z_{i_{j}}-x_{i}\right\rangle>0, \quad j=1,2, \ldots, n
$$

Then we have

$$
0<\sum_{j=1}^{n} \alpha_{j}\left\langle p_{i}, z_{i_{j}}-x_{i}\right\rangle=\left\langle p_{i}, x_{i}-x_{i}\right\rangle=0
$$

a contradiction.

Definition 1.4 [18]. For each $i \in I$, let $E_{i}, Y_{i}$ be two topological vector spaces, $X_{i}$ a nonempty and convex subset of $E_{i}, C_{i}: X \rightarrow 2^{Y_{i}}$ a set-valued map such that $C_{i}(x)$ is a closed, pointed, and convex cone for each $x \in X$. Let $\eta_{i}: X_{i} \times X_{i} \rightarrow E_{i}$ be a single-valued map. $T_{i}: X \rightarrow 2^{L\left(E_{i}, Y_{i}\right)}$ is said to satisfy the generalized partial $L-\eta_{i}$-condition if and only if for any finite set $\left\{y_{i_{1}}, y_{i_{2}}, \ldots, y_{i_{n}}\right\}$ in $X_{i}$, for all $\bar{x}=\left(\bar{x}^{i}, \bar{x}_{i}\right)$ with $\bar{x}_{i}=\sum_{j=1}^{n} \alpha_{j} y_{i_{j}}$, where $\alpha_{j} \geq 0$ and $\sum_{j=1}^{n} \alpha_{j}=1$, there exists $\bar{v}_{i} \in T_{i}(\bar{x})$ such that

$$
\left\langle\bar{v}_{i}, \sum_{j=1}^{n} \alpha_{j} \eta_{i}\left(y_{i_{j}}, \bar{x}_{i}\right)\right\rangle \notin-\operatorname{int} C_{i}(\bar{x}) .
$$


Definition 1.5 [36]. Let $X$ be a nonempty convex subset of a topological vector space $E$ and $P$ a closed, pointed, and convex cone in a topological vector space $Y$. Let $F: X \rightarrow 2^{Y}$ be a set-valued map. Then $F$ is said to be naturally quasiconvex on $X$, if for any $x_{1}, x_{2} \in X$ and $\lambda \in[0,1]$,

$$
F\left(\lambda x_{1}+(1-\lambda) x_{2}\right) \subseteq \operatorname{Co}\left\{F\left(x_{1}\right) \cup F\left(x_{2}\right)\right\}-P .
$$

Definition 1.6 [8]. Let $E$ be a Hausdorff topological space and $L$ a lattice with least element, denoted by 0 . A map $\Phi: 2^{E} \rightarrow L$ is a measure of noncompactness provided that the following conditions hold for all $M, N \in 2^{E}$ :

(i) $\Phi(M)=0$ if and only if $M$ is precompact (i.e., it is relatively compact);

(ii) $\Phi(\overline{\operatorname{conv}} M)=\Phi(M)$; where $\overline{\operatorname{conv}} M$ denotes the convex closure of $M$;

(iii) $\Phi(M \cup N)=\max \{\Phi(M), \Phi(N)\}$.

Definition 1.7 [8]. Let $\Phi: 2^{E} \rightarrow L$ be a measure of noncompactness on $E$ and $D \subseteq E$. A setvalued map $Q: D \rightarrow 2^{E}$ is called $\Phi$-condensing provided that if $M \subseteq D$ with $\Phi(Q(M)) \geq$ $\Phi(M)$, then $M$ is relatively compact.

It is clear that if $Q: D \rightarrow 2^{E}$ is $\Phi$-condensing and $Q^{*}: D \rightarrow 2^{E}$ satisfies $Q^{*}(x) \subseteq Q(x)$ for all $x \in D$, then $Q^{*}$ is also $\Phi$-condensing.

In the next section, we will use the following particular form of a maximal element theorem for a family of set-valued maps due to Deguire et al. [33, Theorem 7] (also see [20, Theorem 1]).

LEMma 1.8. Let I be any index set and $\left\{X_{i}\right\}_{i \in I}$ a family of nonempty convex subsets where each $X_{i}$ is contained in a Hausdorff topological vector space $E_{i}$. For each $i \in I$, let $S_{i}: X \rightarrow 2^{X_{i}}$ be a set-valued map such that

(i) $S_{i}(x)$ is convex,

(ii) for each $x \in X, x_{i} \notin S_{i}(x)$,

(iii) for each $y_{i} \in X_{i}, S_{i}^{-1}\left(y_{i}\right)$ is open in $X$,

(iv) there exist a nonempty compact subset $N$ of $X$ and a nonempty compact convex subset $B_{i}$ of $X_{i}$ for each $i \in I$ such that for each $x \in X \backslash N$ there exists $i \in I$ satisfying $S_{i}(x) \cap$ $B_{i} \neq \varnothing$. Then there exists $\bar{x} \in X$ such that $S_{i}(\bar{x})=\varnothing$ for all $i \in I$.

The following lemma is a particular form of [35, Corollary 4].

Lemma 1.9 (maximal element theorem). Let I be any index set and $\left\{X_{i}\right\}_{i \in I}$ a family of nonempty, closed, and convex subsets where each $X_{i}$ is contained in a locally convex Hausdorff topological vector space $E_{i}$. For each $i \in I$, let $S_{i}: X \rightarrow 2^{X_{i}}$ be a set-valued map such that

(i) for each $x \in X, x_{i} \notin \operatorname{CoS}_{i}(x)$,

(ii) for each $y_{i} \in X_{i}, S_{i}^{-1}\left(y_{i}\right)$ is open in $X$,

(iii) the set-valued map $S: X \rightarrow 2^{X}$ defined as $S(x)=\prod_{i \in I} S_{i}(x)$, for all $x \in X$, is $\Phi$ condensing.

Then there exists $\bar{x} \in X$ such that $S_{i}(x)=\varnothing$ for all $i \in I$.

Lemma 1.10 [34]. Let $X$ and $Y$ be topological spaces and $T: X \rightarrow 2^{Y}$ be an upper semicontinuous set-valued map with compact values. Suppose $\left\{x_{\alpha}\right\}$ is a net in $X$ such that $x_{\alpha} \rightarrow x_{0}$. 
If $y_{\alpha} \in T\left(x_{\alpha}\right)$ for each $\alpha$, then there is a $y_{0} \in T\left(x_{0}\right)$ and a subset $\left\{y_{\beta}\right\}$ of $\left\{y_{\alpha}\right\}$ such that $y_{\beta} \rightarrow y_{0}$.

\section{Existence results}

In this section, we present some existence results of a solution for the SGVQEP and its special cases without or with $\Phi$-condensing maps.

Theorem 2.1. Let $I$ be any index set. For each $i \in I$, let $Y_{i}$ be a topological vector space and $X_{i}$ a nonempty convex set in a Hausdorff topological vector space $E_{i}$, let $F_{i}: X \times X_{i} \rightarrow 2^{Y_{i}}$ be a set-valued map, and let $D_{i}: X \rightarrow 2^{X_{i}}$ be a set-valued map such that $D_{i}(x)$ is nonempty and convex for all $x \in X, D_{i}^{-1}\left(y_{i}\right)$ is open in $X$ for all $y_{i} \in X_{i}$, and the set $W_{i}=\{x \in X$ : $\left.x_{i} \in D_{i}(x)\right\}$ is closed in $X$. Let $C_{i}: X \rightarrow 2^{Y_{i}}$ be a set-valued map such that $C_{i}(x)$ is a convex, pointed, and closed cone with int $C_{i}(x) \neq \varnothing$ for all $x \in X$. Assume that the following conditions are satisfied:

(i) for each $i \in I, F_{i}$ is $C_{i-x}-0$-partially diagonal quasiconvex;

(ii) for each $i \in I$, for each $y_{i} \in X_{i}, F_{i}\left(\cdot, y_{i}\right)$ is upper semicontinuous on $X$ with compact values;

(iii) for each $i \in I$, the set-valued map - int $C_{i}$ has open graph in $X \times Y_{i}$;

(iv) there exist a nonempty and compact subset $N$ of $X$ and a nonempty, compact, and convex subset $B_{i}$ of $X_{i}$ for each $i \in I$ such that for all $x=\left(x^{i}, x_{i}\right) \in X \backslash N$, there exist $i \in I$ and $\bar{y}_{i} \in B_{i}$, such that $\bar{y}_{i} \in D_{i}(x)$ and $F_{i}\left(x, \bar{y}_{i}\right) \subseteq-\operatorname{int} C_{i}(x)$.

Then, the solution set of the SGVQEP is nonempty.

Proof. For each $i \in I$, we define a set-valued map $P_{i}: X \rightarrow 2^{X_{i}}$ by

$$
P_{i}(x)=\left\{y_{i} \in X_{i}: F_{i}\left(x, y_{i}\right) \subseteq-\operatorname{int} C_{i}(x)\right\}, \quad \forall x=\left(x^{i}, x_{i}\right) \in X
$$

By hypothesis (i), we have $x_{i} \notin \operatorname{Co} P_{i}(x)$ for each $i \in I$ and for all $x \in X$. To see this, suppose, by way of contradiction, that there exist $i \in I$ and a point $\bar{x}=\left(\bar{x}^{i}, \bar{x}_{i}\right) \in X$ such that $\bar{x}_{i} \in \operatorname{Co} P_{i}(\bar{x})$. Then there exist finite points $y_{i_{1}}, y_{i_{2}}, \ldots, y_{i_{n}}$ in $X_{i}$, and $\lambda_{j} \geq 0$ with $\sum_{j=1}^{n} \lambda_{j}=$ 1 such that $\bar{x}_{i}=\sum_{j=1}^{n} \lambda_{j} y_{i_{j}}$ and $y_{i_{j}} \in P_{i}(\bar{x})$ for all $j=1,2, \ldots, n$. That is, $F_{i}\left(\bar{x}, y_{i_{j}}\right) \subseteq$ $-\operatorname{int} C_{i}(\bar{x})$ for $j=1,2, \ldots, n$, which contradicts the hypothesis that $F_{i}$ is $C_{i-x}-0$-partially diagonal quasiconvex.

By hypothesis (ii), we can prove that for each $i \in I$, and for each $y_{i} \in X_{i}$, the set $P_{i}^{-1}\left(y_{i}\right)=\left\{x \in X: F_{i}\left(x, y_{i}\right) \subseteq-\operatorname{int} C_{i}(x)\right\}$ is open, that is, $P_{i}$ has open lower sections. Let $Q_{i}\left(y_{i}\right)=\left\{x \in X: F_{i}\left(x, y_{i}\right) \nsubseteq-\operatorname{int} C_{i}(x)\right\}$ for all $y_{i} \in X_{i}$. We can prove that $Q_{i}\left(y_{i}\right)=\{x \in$ $\left.X: F_{i}\left(x, y_{i}\right) \nsubseteq-\operatorname{int} C_{i}(x)\right\}=X \backslash P_{i}^{-1}\left(y_{i}\right)$ is closed for all $y_{i} \in X_{i}$. In fact, consider a net $x_{t} \in X$ such that $x_{t} \rightarrow x \in X$. For every $t$ there exists $u_{t} \in F_{i}\left(x_{t}, y_{i}\right)$ with $u_{t} \notin-\operatorname{int} C_{i}\left(x_{t}\right)$. Since $F_{i}\left(\cdot, y_{i}\right)$ is upper semicontinuous with compact values, by Lemma 1.10, it suffices to find a subset $\left\{u_{t_{j}}\right\}$ which converges to some $u \in F_{i}\left(x, y_{i}\right)$; then $\left(x_{t_{j}}, u_{t_{j}}\right) \rightarrow(x, u)$. It follows that $u \notin-\operatorname{int} C_{i}(x)$ since the graph of $-\operatorname{int} C_{i}(\cdot)$ is open, hence, $x \in Q_{i}\left(y_{i}\right)$.

So we have proved that $P_{i}$ has open lower sections. Then by [4, Lemma 5], we know that the set-valued map $\operatorname{Co} P_{i}: X \rightarrow 2^{X_{i}}$ defined by $\operatorname{Co} P_{i}(x)=\operatorname{Co}\left(P_{i}(x)\right)$, for all $x \in X$ also has open lower sections. For each $i \in I$, we also define another set-valued map $S_{i}: X \rightarrow 2^{X_{i}}$ 
by

$$
S_{i}(x)= \begin{cases}D_{i}(x) \cap \operatorname{Co} P_{i}(x) & \text { if } x \in W_{i}, \\ D_{i}(x) & \text { if } x \notin W_{i} .\end{cases}
$$

Then, for each $i \in I$, it is clear that $S_{i}(x)$ is convex for all $x \in X$ and $x_{i} \notin S_{i}(x)$. Since

$$
\begin{aligned}
\forall i \in I & , \forall y_{i} \in X_{i}, \quad S_{i}^{-1}\left(y_{i}\right) \\
= & \left\{x \in X: y_{i} \in S_{i}(x)\right\} \\
= & \left\{x \in W_{i}: y_{i} \in D_{i}(x) \cap \operatorname{Co} P_{i}(x)\right\} \cup\left\{x \in X \backslash W_{i}: y_{i} \in D_{i}(x)\right\} \\
= & \left(W_{i} \cap D_{i}^{-1}\left(y_{i}\right) \cap \operatorname{Co} P^{-1}\left(y_{i}\right)\right) \cup\left[\left(X \backslash W_{i}\right) \cap D^{-1}\left(y_{i}\right)\right] \\
= & {\left[\left(W_{i} \cap D_{i}^{-1}\left(y_{i}\right) \cap \operatorname{Co} P_{i}^{-1}\left(y_{i}\right)\right) \cup\left(X \backslash W_{i}\right)\right] } \\
& \cap\left[\left(W_{i} \cap D_{i}^{-1}\left(y_{i}\right) \cap \operatorname{Co} P_{i}^{-1}\left(y_{i}\right)\right) \cup D_{i}^{-1}\left(y_{i}\right)\right] \\
= & \left\{X \cap\left[\left(D_{i}^{-1}\left(y_{i}\right) \cap \operatorname{Co} P_{i}^{-1}\left(y_{i}\right)\right) \cup\left(X \backslash W_{i}\right)\right]\right\} \cap\left[\left(W_{i} \cup D_{i}^{-1}\left(y_{i}\right)\right) \cap\left(D_{i}^{-1}\left(y_{i}\right)\right)\right] \\
= & {\left[\left(D_{i}^{-1}\left(y_{i}\right) \cap \operatorname{Co} P_{i}^{-1}\left(y_{i}\right)\right) \cup\left(X \backslash W_{i}\right)\right] \cap D_{i}^{-1}\left(y_{i}\right) } \\
= & \left(D_{i}^{-1}\left(y_{i}\right) \cap\left(\operatorname{Co} P_{i}^{-1}\left(y_{i}\right)\right) \cup\left(\left(X \backslash W_{i}\right) \cap\left(D_{i}^{-1}\left(y_{i}\right)\right)\right),\right.
\end{aligned}
$$

and $D_{i}^{-1}\left(y_{i}\right), \operatorname{Co} P_{i}^{-1}\left(y_{i}\right)$ and $X \backslash W_{i}$ are open in $X$, we have $S_{i}^{-1}\left(y_{i}\right)$ open in $X$.

By assumption (iv), we know that the condition (iv) of Lemma 1.8 holds. Hence, by Lemma 1.8, there exists $\bar{x} \in X$ such that $S_{i}(\bar{x})=\varnothing$, for all $i \in I$. Since for all $i \in I$ and for all $x \in X, D_{i}(x)$ is nonempty, we have $\bar{x} \in W_{i}$, and $D_{i}(\bar{x}) \cap \operatorname{Co} P_{i}(\bar{x})=\varnothing$, for all $i \in I$. This implies $\bar{x}_{i} \in D_{i}(\bar{x})$ and $D_{i}(\bar{x}) \cap P_{i}(\bar{x})=\varnothing$, for all $i \in I$. Therefore, for all $i \in I$,

$$
\bar{x}_{i} \in D_{i}(\bar{x}), \quad F_{i}\left(\bar{x}, y_{i}\right) \nsubseteq-\operatorname{int} C_{i}(\bar{x}), \quad \forall y_{i} \in D_{i}(\bar{x}) .
$$

That is, the solution set of the SGVQEP is nonempty. This completes the proof.

Corollary 2.2. If we replace, in Theorem 2.1, condition (i) by the following conditions;

(a) for each $i \in I$, for all $x \in X, y_{i} \mapsto F_{i}\left(x, y_{i}\right)$ is natural $P_{i}$-quasiconvex;

(b) for each $i \in I$, for all $x \in X, F_{i}\left(x, x_{i}\right) \nsubseteq-\operatorname{int} C_{i}(x)$;

then the conclusion of Theorem 2.1 still holds, that is, the solution set of the SGVQEP is nonempty.

Proof. For each $i \in I$, we define a set-valued map $P_{i}: X \rightarrow 2^{X_{i}}$ by

$$
P_{i}(x)=\left\{y_{i} \in X_{i}: F_{i}\left(x, y_{i}\right) \subseteq-\operatorname{int} C_{i}(x)\right\}, \quad \forall x=\left(x^{i}, x_{i}\right) \in X .
$$

Then $P_{i}(x)$ is convex for each $i \in I$ and for all $x \in X$.

To prove it, we fix arbitrary $i \in I$ and $x \in X$. Let $y_{i_{1}}, y_{i_{2}} \in P_{i}(x)$ and $\lambda \in[0,1]$, then we have

$$
F_{i}\left(x, y_{i_{j}}\right) \subseteq-\operatorname{int} C_{i}(x), \quad j=1,2 .
$$


By the convexity of $-\operatorname{int} C_{i}(x)$, we have

$$
\operatorname{Co}\left\{F_{i}\left(x, y_{i_{1}}\right) \cup F_{i}\left(x, y_{i_{2}}\right)\right\} \subseteq-\operatorname{int} C_{i}(x) \text {. }
$$

Since $F_{i}$ is natural $P_{i}$-quasiconvex,

$$
F_{i}\left(x, \lambda y_{i_{1}}+(1-\lambda) y_{i_{2}}\right) \subseteq \operatorname{Co}\left\{F_{i}\left(x, y_{i_{1}}\right) \cup F_{i}\left(x, y_{i_{2}}\right)\right\}-P_{i} \subseteq-\operatorname{int} C_{i}(x)-P_{i} \subseteq-\operatorname{int} C_{i}(x) .
$$

Hence, $\lambda y_{i_{1}}+(1-\lambda) y_{i_{2}} \in P_{i}(x)$ and so $P_{i}(x)$ is convex.

We show that $F_{i}$ is $C_{i-x}-0$-partially diagonal quasiconvex for each $i \in I$. If not, then there exists a finite subset $\left\{y_{i_{1}}, y_{i_{2}}, \ldots, y_{i_{n}}\right\}$ in $X_{i}$, and a point $x=\left(x^{i}, x_{i}\right) \in X$ with $x_{i} \in$ $\operatorname{Co}\left\{y_{i_{1}}, y_{i_{2}}, \ldots, y_{i_{n}}\right\}$ such that

$$
F_{i}\left(x, y_{i_{j}}\right) \subseteq-\operatorname{int} C_{i}(x), \quad j=1,2, \ldots, n
$$

Since $P_{i}(x)=\left\{y_{i} \in X_{i}: F_{i}\left(x, y_{i}\right) \subseteq-\operatorname{int} C_{i}(x)\right\}$ is a convex set, $x_{i} \in P_{i}(x)$, that is, $F_{i}\left(x, x_{i}\right) \subseteq$ $-\operatorname{int} C_{i}(x)$, which contradicts the condition (b).

By Theorem 2.1, we know the conclusion of Corollary 2.2 holds. This completes the proof.

Corollary 2.3. If we replace, in Theorem 2.1, condition (i) by the following conditions:

(a) for each $i \in I, F_{i}$ is $C_{i}(x)$-quasiconvex-like;

(b) for each $i \in I$, for all $x \in X, F_{i}\left(x, x_{i}\right) \nsubseteq-\operatorname{int} C_{i}(x)$;

then the conclusion of Theorem 2.1 still holds, that is, the solution set of the (SGVQEP) is nonempty.

Proof. For each $i \in I$, let the set-valued map $P_{i}: X \rightarrow 2^{X_{i}}$ be defined the same as that in the proof of Corollary 2.2. Then by the assumption (a) and the proof of [20, Theorem $3]$, it is easy to see that for all $i \in I$ and for all $x \in X, P_{i}(x)$ is convex. The conclusion of Corollary 2.3 follows from the Corollary 2.2. This completes the proof.

Remark 2.4. If $D_{i}(x)=X_{i}$ for all $x \in X$ and for all $i \in I$, then by Corollary 2.3, we recover [20, Theorem 3]. Hence, Theorem 2.1 generalizes [20, Theorem 3] from the system of generalized vector equilibrium problems to the system of generalized vector quasiequilibrium problems with more general convex conditions.

Remark 2.5. If $F_{i}$ is replaced by a single-valued map $f_{i}: X \times X_{i} \rightarrow Y_{i}$, then by [21, Remark 5(1) and Corollary 2.2], we can obtain [21, Theorem 2]. Hence, Theorem 2.1 generalizes [21, Theorem 2] from single-valued case to set-valued case with more general convex conditions.

Remark 2.6. For each $i \in I$, let the set-valued map $F_{i}$ be replaced by a single-valued map $\varphi_{i}: X \times X_{i} \rightarrow Y_{i}$, and let $C_{i}(x)=C_{i}$ and $D_{i}(x)=X_{i}$ for all $x \in X$. By Theorem 2.1, we have the existence result of the SVEP as follows.

Let $I$ be any index set. For each $i \in I$, let $Y_{i}$ be a topological vector space, $X_{i}$ a nonempty compact convex set in a Hausdorff topological vector space $E_{i}$, and $\varphi_{i}: X \times X_{i} \rightarrow Y_{i}$ a single-valued map. Let $C_{i} \subseteq Y_{i}$ be a convex, pointed, and closed cone with int $C_{i}(x) \neq \varnothing$ 
for all $x \in X$. Assume that the following conditions are satisfied

(i) for each $i \in I, \varphi_{i}$ is $C_{i}-0$-partially diagonal quasiconvex;

(ii) for each $i \in I$, for each $y_{i} \in X_{i}, \varphi_{i}\left(\cdot, y_{i}\right)$ is continuous on $X$.

Then, there exists $\bar{x}=\left(\bar{x}^{i}, \bar{x}_{i}\right)$ in $X$ such that for each $i \in I, \bar{x}_{i} \in X_{i}$ and $\varphi_{i}\left(\bar{x}, y_{i}\right) \notin-\operatorname{int} C_{i}$, for all $y_{i} \in X_{i}$. That is, the solution set of the SVEP is nonempty.

And if the condition (i) of the above result is replaced by $\varphi_{i}\left(x, x_{i}\right)=0$ for all $x \in X$ and the map $y_{i} \mapsto \varphi_{i}\left(x, y_{i}\right)$ is $C_{i}$-quasiconvex, then we recover [19, Theorem 2.1]. If $\varphi_{i}\left(x, x_{i}\right)=$ 0 for all $x \in X$ and the map $y_{i} \mapsto \varphi_{i}\left(x, y_{i}\right)$ is $C_{i}$-quasiconvex, then $\varphi_{i}$ must be $C_{i}$ - 0 -partially diagonal quasiconvex. Hence, Theorem 2.1 generalizes [19, Theorem 2.1] in several aspects.

Now we establish an existence result for a solution to the SGVQEP involving $\Phi$ condensing maps.

TheORem 2.7. Let I be any index set. For each $i \in I$, let $Y_{i}$ be a topological vector space and $X_{i}$ a nonempty, closed, and convex set in a locally convex Hausdorff topological vector space $E_{i}$. Let $F_{i}: X \times X_{i} \rightarrow 2^{Y_{i}}$ be a set-valued map and $D_{i}: X \rightarrow 2^{X_{i}}$ a set-valued map such that for all $x \in X, D_{i}(x)$ is a nonempty convex set, $D_{i}^{-1}\left(y_{i}\right)$ is open in $X$ for all $y_{i} \in X_{i}$, and the set $W_{i}=\left\{x \in X: x_{i} \in D_{i}(x)\right\}$ is closed in $X$. Let $C_{i}: X \rightarrow 2^{Y_{i}}$ be a set-valued map such that $C_{i}(x)$ is a closed, pointed, and convex cone with int $C_{i}(x) \neq \varnothing$ for all $x \in X$. Assume that the set-valued map $D: X \rightarrow 2^{X}$ defined as $D(x)=\prod_{i \in I} D_{i}(x)$, for all $x \in X$, is $\Phi$-condensing and the conditions (i), (ii), and (iii) of Theorem 2.1 hold. Then the solution set of SGVQEP is nonempty.

Proof. In view of Lemma 1.9, it is sufficient to show that the set-valued map $S: X \rightarrow 2^{X}$ defined as $S(x)=\prod_{i \in I} S_{i}(x)$ for all $x \in K$ is $\Phi$-condensing, where $S_{i}$ 's are the same as defined in the proof of Theorem 2.1. By the definition of $S_{i}, S_{i}(x) \subseteq D_{i}(x)$ for all $x \in X$ and for each $i \in I$, and therefore $S(x) \subseteq D(x)$ for all $x \in X$. Since $D$ is $\Phi$-condensing, so is $S$. This completes the proof.

Remark 2.8. Theorem 2.7 generalizes [21, Theorem 3] from single-valued case to setvalued case with more general convex conditions.

Remark 2.9. If we replace, in Theorem 2.7, condition (i) and (ii) of Theorem 2.1, respectively, by the following conditions:

(a) for each $i \in I, F_{i}$ is the second-type $C_{i-x}$-0-partially diagonal quasiconvex;

(b) for each $i \in I$, for each $z_{i} \in X_{i}, F_{i}\left(\cdot, z_{i}\right)$ is lower semicontinuous on $X$; then there exists $\bar{x}=\left(\bar{x}^{i}, \bar{x}_{i}\right)$ in $X$ such that for each $i \in I$,

$$
\bar{x}_{i} \in D_{i}(\bar{x}), \quad F_{i}\left(\bar{x}, z_{i}\right) \subseteq Y_{i} \backslash\left(-\operatorname{int} C_{i}(\bar{x})\right), \quad \forall z_{i} \in D_{i}(\bar{x}) .
$$

This is [32, Theorem 2.1].

We will prove the existence of solutions for the SGVQVLIP as follows.

Corollary 2.10. Let I be any index set. For each $i \in I$, let $Y_{i}$ be a real Hausdorff topological vector space and $X_{i}$ a nonempty convex set in a real Hausdorff topological vector space $E_{i}$. Let the set-valued maps $D_{i}: X \rightarrow 2^{X_{i}}, C_{i}: X \rightarrow 2^{Y_{i}}$, and the set $W_{i}=\left\{x \in X: x_{i} \in D_{i}(x)\right\}$ be as 
in Theorem 2.1, and let $L\left(E_{i}, Y_{i}\right)$ be equipped with the $\sigma$-topology. Assume that the following conditions are satisfied:

(i) for each $i \in I, T_{i}: X \rightarrow 2^{L\left(E_{i}, Y_{i}\right)}$ is an upper semicontinuous set-valued map with nonempty compact values, $\eta_{i}: X_{i} \times X_{i} \rightarrow E_{i}$ is continuous with respect to the second argument, such that $T_{i}$ satisfies the generalized partial $L-\eta_{i}$-condition;

(ii) there exist a nonempty and compact subset $N$ of $X$ and a nonempty, compact, and convex subset $B_{i}$ of $X_{i}$ for each $i \in I$ such that for all $x=\left(x^{i}, x_{i}\right) \in X \backslash N$, there exist $i \in I$ and $\bar{y}_{i} \in B_{i}$, such that $\bar{y}_{i} \in D_{i}(x)$ and $\left\langle v_{i}, \eta_{i}\left(\bar{y}_{i}, x_{i}\right)\right\rangle \in-\operatorname{int} C_{i}(x)$, for all $v_{i} \in$ $T_{i}(x)$.

Then the SGVQVLI has a solution $\bar{x} \in X$.

Proof. For each $i \in I$, define a set-valued map $P_{i}: X \rightarrow 2^{X_{i}}$ by

$$
\begin{aligned}
P_{i}(x) & =\left\{y_{i} \in X_{i}:\left\langle T_{i}(x), \eta_{i}\left(y_{i}, x_{i}\right)\right\rangle \subseteq-\operatorname{int} C_{i}(x)\right\} \\
& =\left\{y_{i} \in X_{i}:\left\langle v_{i}, \eta_{i}\left(y_{i}, x_{i}\right)\right\rangle \in-\operatorname{int} C_{i}(x), \forall v_{i} \in T_{i}(x)\right\}, \quad \forall x \in X .
\end{aligned}
$$

From the proof of [18, Theorem 3.1], we know that $x_{i} \notin \operatorname{Co}\left(P_{i}(x)\right)$ for all $x=\left(x^{i}, x_{i}\right) \in$ $X$ and the set $P_{i}^{-1}\left(y_{i}\right)=\left\{x \in X:\left\langle T_{i}(x), \eta_{i}\left(y_{i}, x_{i}\right)\right\rangle \subseteq-\operatorname{int} C_{i}(x)\right\}$ is open for each $i \in I$ and for each $y_{i} \in X_{i}$. That is, $P_{i}$ has open lower sections in $X$.

The remainder of the proof is same as that in the proof of Theorem 2.1.

In view of Lemma 1.9 and the proof of Corollary 2.10, it is easy to obtain an existence result of a solution to SGVQVLI as follows.

Corollary 2.11. Let I be any index set. For each $i \in I$, let $Y_{i}$ be a real Hausdorff topological vector space and $X_{i}$ a nonempty, closed, and convex set in a real locally convex Hausdorff topological vector space $E_{i}$, let the set-valued maps $D_{i}: X \rightarrow 2^{X_{i}}, D: X \rightarrow 2^{X}, C_{i}: X \rightarrow 2^{Y_{i}}$ and the set $W_{i}=\left\{x \in X: x_{i} \in D_{i}(x)\right\}$ be the same as those in Theorem 2.7. Assume that condition ( $i$ ) of Corollary 2.10 holds. Then the solution set of SGVQVLI is nonempty.

Remark 2.12. Let $I$ be an index set and let $I$ be countable. For each $i \in I$, let $Y_{i}$ be a real Hausdorff topological vector space, let $X_{i}$ be a nonempty, compact, convex, and metrizable set in a real locally convex Hausdorff topological vector space $E_{i}$, let $D_{i}: X \rightarrow 2^{X_{i}}$ be an upper semicontinuous set-valued mapping with nonempty convex closed values and open lower sections, let $C_{i}: X \rightarrow 2^{Y_{i}}$ be a set-valued mapping such that $C_{i}(x)$ is a closed pointed and convex cone with int $C_{i}(x) \neq \varnothing$ for each $x \in X$, and the set-valued map $M_{i}=Y_{i} \backslash\left(-\operatorname{int} C_{i}\right): X \rightarrow 2^{Y_{i}}$ is upper semicontinuous, and let $L\left(E_{i}, Y_{i}\right)$ be equipped with the $\sigma$-topology. Suppose that the condition (i) of Corollary 2.10 satisfied, then the SGVQVLI has a solution $\bar{x} \in X$.

The above is [18, Theorem 3.1]. It is easy to see that Corollaries 2.10 and 2.11 generalize [18, Theorem 3.1] without compactness and metrizability of $X_{i}$ and with weaker conditions of $D_{i}$. Corollaries 2.10 and 2.11 also generalize [20, Corollaries 2 and 3] and [19, Theorems 3.1 and 3.2] in several aspects.

Remark 2.13. By the above results, it is easy to obtain the existence results of a solution for the other special cases of the SGVQEP and they are omitted here. 


\section{Acknowledgments}

This research was supported by the National Natural Science Foundation of China (Grant no. 10171118), the Education Committee Project Research Foundation of Chongqing (Grant no. 030801), and the Natural Science Foundation of Chongqing (no. 8409). The authors would like to express their thanks to the referees for their comments and suggestions that improved the presentation of this manuscript.

\section{References}

[1] J.-P. Aubin, Mathematical Methods of Game and Economic Theory, Studies in Mathematics and Its Applications, vol. 7, North-Holland, Amsterdam, 1979.

[2] J.-P. Aubin and I. Ekeland, Applied Nonlinear Analysis, Pure and Applied Mathematics (New York), John Wiley \& Sons, New York, 1984.

[3] J. X. Zhou and G. Y. Chen, Diagonal convexity conditions for problems in convex analysis and quasi-variational inequalities, J. Math. Anal. Appl. 132 (1988), no. 1, 213-225.

[4] G. Q. Tian and J. X. Zhou, Quasi-variational inequalities without the concavity assumption, J. Math. Anal. Appl. 172 (1993), no. 1, 289-299.

[5] G. X.-Z. Yuan and E. Tarafdar, Generalized quasi-variational inequalities and some applications, Nonlinear Anal. 29 (1997), no. 1, 27-40.

[6] L. J. Lin and S. Park, On some generalized quasi-equilibrium problems, J. Math. Anal. Appl. 224 (1998), no. 2, 167-181.

[7] S. Park, On minimax inequalities on spaces having certain contractible subsets, Bull. Austral. Math. Soc. 47 (1993), no. 1, 25-40.

[8] P. M. Fitzpatrick and W. V. Petryshyn, Fixed point theorems for multivalued noncompact acyclic mappings, Pacific J. Math. 54 (1974), no. 2, 17-23.

[9] E. Michael, Continuous selections. I, Ann. of Math. (2) 63 (1956), 361-382.

[10] W. Shafer and H. Sonnenschein, Equilibrium in abstract economies without ordered preferences, J. Math. Econom. 2 (1975), no. 3, 345-348.

[11] U. Mosco, Implicit variational problems and quasi variational inequalities, Nonlinear Operators and the Calculus of Variations (Summer School, Univ. Libre Bruxelles, Brussels, 1975), Lecture Notes in Math., vol. 543, Springer, Berlin, 1976, pp. 83-156.

[12] Q. H. Ansari and F. Flores-Bazán, Generalized vector quasi-equilibrium problems with applications, J. Math. Anal. Appl. 277 (2003), no. 1, 246-256.

[13] J.-Y. Fu, Generalized vector quasi-equilibrium problems, Math. Methods Oper. Res. 52 (2000), no. $1,57-64$.

[14] Y. H. Cheng, G. Y. Chen, and D. L. Zhu, Existence of solutions of strong vector quasi-equilibrium problems, OR Trans. 11 (2001), no. 4, 9-14.

[15] J. W. Peng, Generalized vectorial quasi-equilibrium problem on $W$-space, J. Math. Res. Exposition 22 (2002), no. 4, 519-524.

[16] J.-S. Pang, Asymmetric variational inequality problems over product sets: applications and iterative methods, Math. Programming 31 (1985), no. 2, 206-219.

[17] E. Allevi, A. Gnudi, and I. V. Konnov, Generalized vector variational inequalities over product sets, Nonlinear Anal. 47 (2001), no. 1, 573-582.

[18] J. W. Peng, System of generalised set-valued quasi-variational-like inequalities, Bull. Austral. Math. Soc. 68 (2003), no. 3, 501-515.

[19] Q. H. Ansari, S. Schaible, and J. C. Yao, System of vector equilibrium problems and its applications, J. Optim. Theory Appl. 107 (2000), no. 3, 547-557.

[20] _ The system of generalized vector equilibrium problems with applications, J. Global Optim. 22 (2002), no. 1-4, 3-16. 
[21] Q. H. Ansari, W. K. Chan, and X. Q. Yang, The system of vector quasi-equilibrium problems with applications, J. Global Optim. 29 (2004), no. 1, 45-57.

[22] C. Ionescu Tulcea, On the approximation of upper semi-continuous correspondences and the equilibriums of generalized games, J. Math. Anal. Appl. 136 (1988), no. 1, 267-289.

[23] G. X.-Z. Yuan, G. Isac, K.-K. Tan, and J. Yu, The study of minimax inequalities, abstract economics and applications to variational inequalities and Nash equilibria, Acta Appl. Math. $\mathbf{5 4}$ (1998), no. 2, 135-166.

[24] G. Cohen and F. Chaplais, Nested monotony for variational inequalities over product of spaces and convergence of iterative algorithms, J. Optim. Theory Appl. 59 (1988), no. 3, 369-390.

[25] M. Bianchi, Pseudo P-monotone operators and variational inequalities, Tech. Rep. 6, Istituto di Econometria e Matematica per le Decisioni Economiche, Università Cattolica del Sacro Cuore, Milan, 1993.

[26] Q. H. Ansari and J. C. Yao, A fixed point theorem and its applications to a system of variational inequalities, Bull. Austral. Math. Soc. 59 (1999), no. 3, 433-442.

[27] L. J. Lin, Z. T. Yu, and G. Kassay, Existence of equilibria for multivalued mappings and its application to vectorial equilibria, J. Optim. Theory Appl. 114 (2002), no. 1, 189-208.

[28] W. Oettli and D. Schläger, Existence of equilibria for monotone multivalued mappings, Math. Methods Oper. Res. 48 (1998), no. 2, 219-228.

[29] Q. H. Ansari, I. V. Konnov, and J. C. Yao, On generalized vector equilibrium problems, Nonlinear Anal. 47 (2001), no. 1, 543-554.

[30] G. Y. Chen and H. Yu, Existence of solutions to a random equilibrium system, J. Systems Sci. Math. Sci. 22 (2002), no. 3, 278-284 (Chinese).

[31] X. P. Ding and E. Tarafdar, Generalized vector variational-like inequalities without monotonicity, Vector Variational Inequalities and Vector Equilibria: Mathematical Theories (F. Giannessi, ed.), Nonconvex Optim. Appl., vol. 38, Kluwer Academic, Dordrecht, 2000, pp. 113-124.

[32] J. W. Peng and X. M. Yang, System of generalized vector quasi-equilibrium problems with lower semicontinuous set-valued maps, Proceedings of The 6th International Conference on Optimization: Techniques and Applications, University of Ballarat, Victoria, 2004.

[33] P. Deguire, K. K. Tan, and G. X.-Z. Yuan, The study of maximal elements, fixed points for $L_{S^{-}}$ majorized mappings and their applications to minimax and variational inequalities in product topological spaces, Nonlinear Anal. Ser. A: Theory Methods 37 (1999), no. 7, 933-951.

[34] C. H. Su and V. M. Sehgal, Some fixed point theorems for condensing multifunctions in locally convex spaces, Proc. Amer. Math. Soc. 50 (1975), 150-154, Proceedings of the National Academy of Sciences of the U.S.A.

[35] S. Chebbi and M. Florenzano, Maximal elements and equilibria for condensing correspondences, Nonlinear Anal. Ser. A: Theory Methods 38 (1999), no. 8, 995-1002.

[36] S. J. Li, G. Y. Chen, and G. M. Lee, Minimax theorems for set-valued mappings, J. Optim. Theory Appl. 106 (2000), no. 1, 183-200.

Jianwen Peng: College of Mathematics and Computer Science, Chongqing Normal University, Chongqing 400047, China

E-mail address: jwpeng6@yahoo.com.cn

Xinmin Yang: College of Mathematics and Computer Science, Chongqing Normal University, Chongqing 400047, China

E-mail address: maxmyang@polyu.edu.cn 


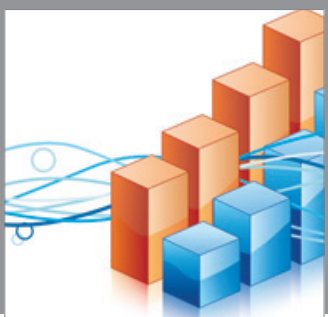

Advances in

Operations Research

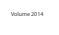

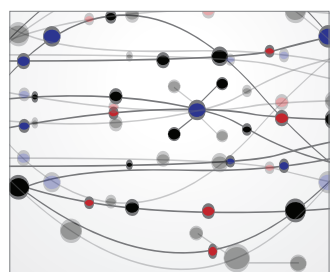

\section{The Scientific} World Journal
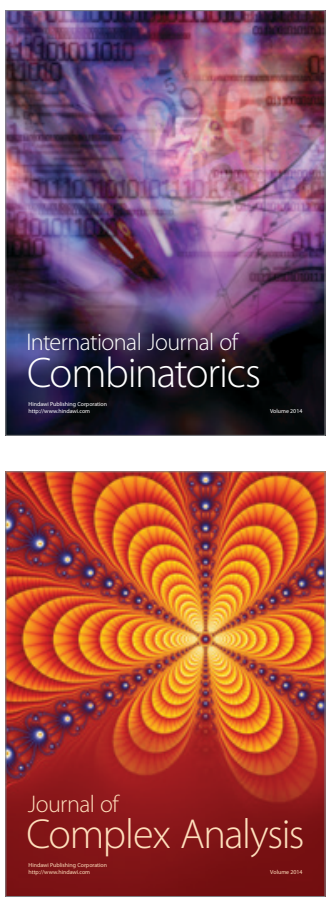

International Journal of

Mathematics and

Mathematical

Sciences
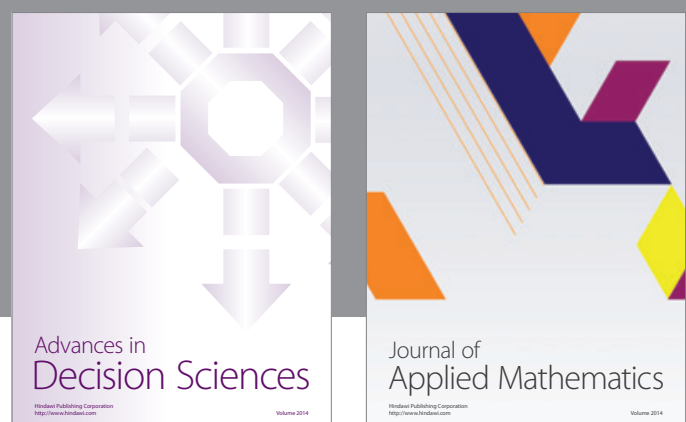

Journal of

Applied Mathematics
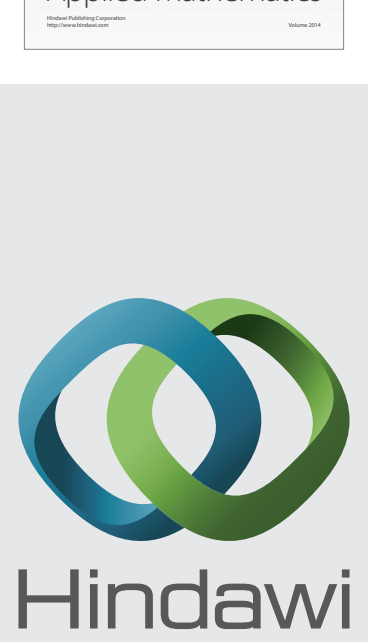

Submit your manuscripts at http://www.hindawi.com
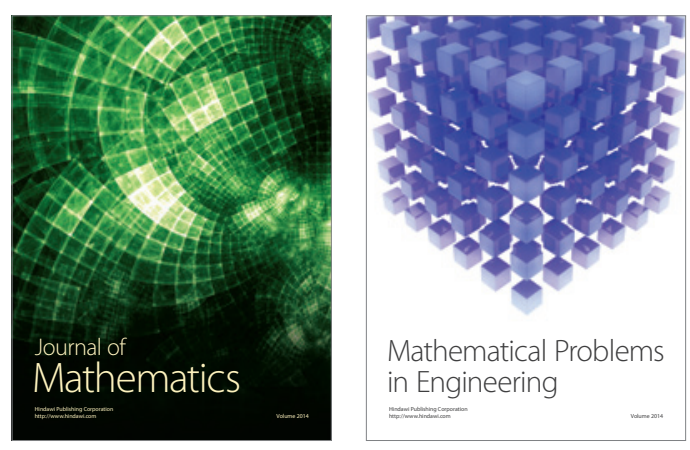

Mathematical Problems in Engineering
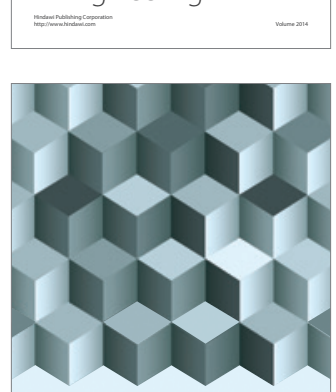

Journal of

Function Spaces
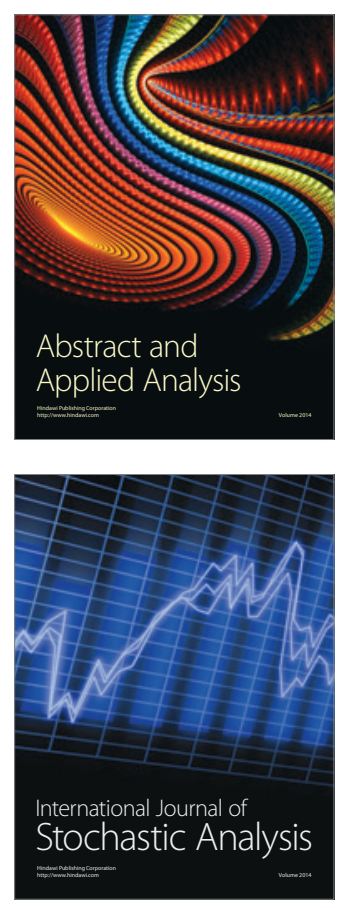

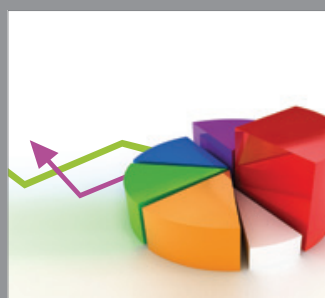

ournal of

Probability and Statistics

Promensencen
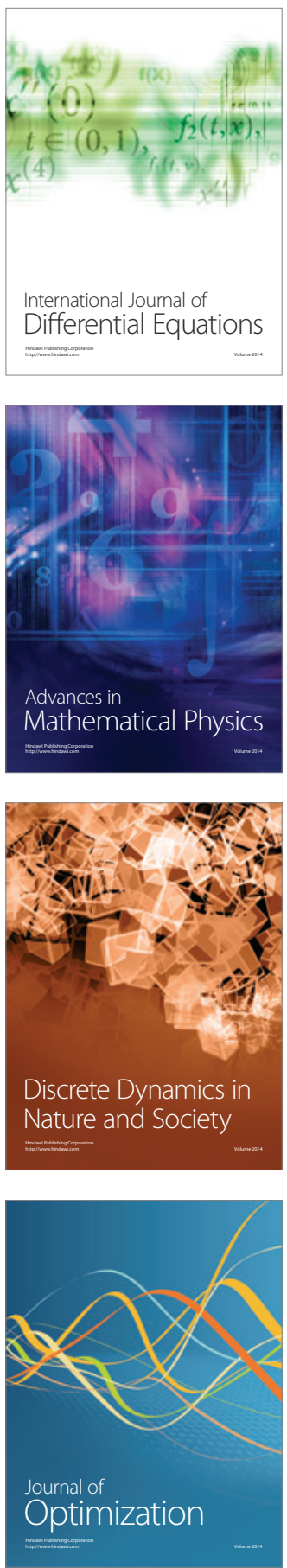